\title{
Testicular Metastasis in a Case of Squamous Cell Carcinoma of the Lung
}

\author{
Dennis Andrew Buck ${ }^{a}$ Robert H. Byrd ${ }^{b} \quad$ Cynthia L. Holmes ${ }^{b}$ \\ Theodore Pollock ${ }^{\mathrm{a}}$ \\ ${ }^{a}$ Southwestern Regional Medical Center, Inc., Cancer Treatment Centers of America, and \\ ${ }^{\mathrm{b}}$ Tulsa Medical Laboratory, Tulsa, Okla., USA
}

\section{Key Words}

Testicular cancer - Testicular metastasis · Blood-testis barrier · Squamous cell · Lung cancer . Sanctuary site

\begin{abstract}
Testicular metastases are a rare finding. Typically, a testicular metastasis will present as a complication of progressive disease or as a primary sign of malignancy. Excluding lymphoma and leukemia, prostate cancer is the most common primary site that metastasizes to the testes. Testicular metastases resulting from squamous cell carcinoma of the lung have rarely been reported in previous literature. Here, we report a case of stage IV squamous cell carcinoma of the lung with metastases to the brain and multiple bilateral lung lesions. Following palliative systemic chemotherapy, this patient was found to have a right testicular mass. Pathology reports confirmed that this was a testicular metastasis of squamous cell lung carcinoma origin.

(c) 2015 S. Karger AG, Basel
\end{abstract}

\section{Introduction}

Testicular neoplasms account for only $1 \%$ of all malignancies. However, testicular germ cell tumors are the most common solid tumors in men between the second and fourth decade of life [1]. Over $90 \%$ of testicular cancers are germ cell tumors that arise from the epithelium of the mature testis. Gonadal stromal tumors derived from cells that play a role in the development and maturation of sperm comprise approximately $5 \%$ of testicular tumors [2]. With the exception of lymphoma and leukemia cases, testicular metastases are extremely 
rare, and the majority of testicular neoplasms are primary tumors [3]. The most frequent primary site that metastasizes to the testes is the prostate followed by lung [4]. However, there are few reports of testicular metastasis originating from squamous cell carcinoma of the lung $[2,5]$. Thus, in order to further the understanding of testicular metastasis and discuss the testis as a known sanctuary site, we present a case of testicular metastasis from squamous cell carcinoma of the lung.

\section{Clinical Findings}

A 58-year-old male who was a former smoker presented to an outside facility complaining of severe headaches. A cranial CT scan revealed multiple CNS metastases in bilateral hemispheres of the brain with the largest one in the right frontal lobe measuring $3.2 \times 3.1$ $\mathrm{cm}$. A CT scan of the chest, abdomen, and pelvis revealed multiple bilateral intrapulmonary nodules with a dominant right upper lobe lesion measuring $3.3 \times 3.1 \times 2.3 \mathrm{~cm}$. A transthoracic needle aspiration biopsy was performed and interpreted as poorly differentiated adenocarcinoma. EGFR mutation and ALK rearrangement analyses were negative. Subsequently, the patient received whole-brain radiation therapy consisting of 37.5 Gy over 15 fractions.

Upon presenting to our facility, a pathologic slide review consultation was performed which was in disagreement with the original diagnosis of poorly differentiated adenocarcinoma and in favor of squamous differentiation due to the morphologic presence of intercellular bridges within cell nests. Bronchoscopy was performed with transbronchial lung biopsy to obtain tissue for further histologic and genetic studies. In contrast to the original diagnosis, this biopsy also revealed poorly differentiated non-small-cell carcinoma in favor of squamous cell carcinoma. Immunohistochemistry performed on the tumor was positive for cytokeratin 7, focally positive for p63, and negative for lung adenocarcinoma markers TTF-1 and napsin-A. Although keratinization was present, intercellular bridges were identified in some of the cell nests, which is morphologic evidence of squamous differentiation. Insufficient tissue was available for ROS1 gene rearrangement analysis by FISH and KRAS mutation analysis by PCR. PET scan results showed extensive pulmonary disease, right frontal lobe brain metastases with increased glycolytic activity, and physiologic uptake in the testicles. The patient was treated with palliative systemic chemotherapy consisting of carboplatin and paclitaxel.

Upon follow-up evaluation, just prior to the third cycle of palliative systemic chemotherapy, the patient presented with an incidental new finding of a palpable lesion on his right testicle. He noticed the mass after viewing a television program discussing the importance of testicular screening, namely palpation and scrotal transillumination. Physical examination revealed a painless, small, palpable mass in the right testis of approximately $1.5 \mathrm{~cm}$ that did not transilluminate. The prostate gland was normal in consistency and size. The contralateral testis and epididymis did not have any palpable masses or irregular consistencies. Both beta-hCG and alpha-fetoprotein were within normal limits as were other pertinent laboratory values. An ultrasound of the right testicle revealed a solid hypervascular mass measuring $1.7 \times 1.5 \times 1.3 \mathrm{~cm}$ in the superolateral region, a small hydrocele, normal flow, and epididymis. The left testicle had a normal anatomy with the exception of a small hydrocele. Upon radiographic review of the CT scan, no abnormalities were found in the retroperitoneal region or pelvic cavity.

A pelvic CT scan identified a testicular tumor suspicious for either a second primary, namely a seminoma, or for a squamous cell lung metastasis. Due to the radiographic findings, physical examination, and out of concern for progressive symptoms such as scrotal 
Buck et al.: Testicular Metastasis in a Case of Squamous Cell Carcinoma of the Lung

pain, the patient was referred for surgical evaluation and a palliative right radical orchiectomy was performed. Pathology of the orchiectomy revealed a well-circumscribed tumor measuring $1.5 \mathrm{~cm}$ with negative surgical margins and no involvement of the spermatic cord. The tumor was composed of irregularly infiltrating and anastomosing nests of large cells with vesicular chromatin, large nucleoli, and abundant cytoplasm (fig. 1), similar to the tumor in the lung biopsy. Immunohistochemistry was positive for squamous cell carcinoma markers cytokeratin 5/6 and p63, while lung adenocarcinoma markers TTF-1 and napsin-A were negative. This pattern supported the diagnosis of metastatic squamous cell carcinoma rather than a new testicular primary (fig. 2).

Further imaging with a CT scan of the chest, abdomen, and pelvis showed enlarging pulmonary lesions indicative of progressive disease. MRI of the brain showed that the brain lesions had a partial response to therapy. Consequently, the patient's treatment regimen was changed to single-agent gemcitabine. Consent was obtained from our patient before this case report was compiled.

\section{Discussion}

Testicular metastases are particularly rare. In a large retrospective study of 738 autopsies of adult males with solid malignancies, only $5(0.68 \%)$ were shown to have testicular metastases [6]. Its incidence among all testicular tumors varies between 0.8 and $2.3 \%$ [3]. Patel et al. [5] reported 209 cases of testicular metastases that were primarily identified from an autopsy series and prostate cancer cases from patients who underwent orchiectomy for hormonal therapy. Out of the 209 cases reviewed, only 13 (6\%) presented with a testicular tumor, and none of these patients had lung cancer. In these cases, prostate tumors were the most common primary (34.6\%) followed by lung carcinomas (17.3\%) [5].

The reason why testicular metastases are so rare is unknown [7]. Several metastasis routes have been postulated for the testes including direct invasion from the adjacent lesions, retrograde venous embolism, arterial embolization, retrograde lymphatic extension from para-aortic lymph nodes, transperitoneal seeding through a congenital hydrocele, or retrograde extension from the vas deferens [8]. Upon disseminating to the testicle, the lower temperature of the intrascrotal contents may impede the ability of the metastatic tumors to become established [7]. The metastatic process consists of several steps. It requires the invasion from the primary tumor, intravasation, survival, arrest and extravasation out of the circulatory system, and colonization of a distant site [9].

Additionally, tumor cells may develop the capacity to preferentially colonize particular organs. Theoretically, organ-specific metastasis may result from a variety of mechanisms. First, tumor cell trapping may occur due to tumor cell arrest based on the pattern of blood flow from the primary tumor to the first capillary bed and the ability of the tumor cells to form aggregates. Second, specific adhesive interactions may occur between tumor cells and the endothelia of certain organs. Lastly, the microenvironment of a distant organ may generate tumor cell niches, or a permissive site for metastatic cells to colonize [9].

Certain differences exist among highly aggressive tumors. In 2006, Tummala et al. [10] reviewed several cases of treatment failure after the completion of adjuvant chemotherapy where a metastasis to the testis was identified. These cases included acute lymphoblastic leukemia, Merkel cell carcinoma, and small-cell carcinoma [7]. Because of these cases, it was proposed that the discovery of a tumor in one testis soon after the completion of adjuvant chemotherapy may suggest that the testes act as a sanctuary site for cancer cells, as chemotherapy effectively eradicated the tumors everywhere else [10]. 
Buck et al.: Testicular Metastasis in a Case of Squamous Cell Carcinoma of the Lung

There are several clinical observations that support the existence of a blood-testis barrier. During puberty, spermatogenesis results in the expression of novel cell-surface antigens after the immune system has developed the ability to recognize foreign cells [11]. However, sperm located within the testis do not elicit an immune response [11]. In addition, although antigen-presenting cells are frequently found within the interstitial space of the testis, macrophages and lymphocytes are rarely found within the seminiferous tubules [11]. Thus, these observations suggest that the testicles may serve as an immune-privileged site [11]. Furthermore, chemotherapy drugs achieve lower concentrations within the interstitium of the testis compared with plasma levels, which suggests that a filtering barrier may exist between the capillaries and the interstitial space [12].

In summary, potential mechanisms of how testicular metastases develop have been well documented. Moreover, the testicles may serve as an immune-privileged region and receive lower concentrations of chemotherapy compared to that of plasma, which allows for metastases to evade therapy. Our case presentation emphasizes the importance of recognizing that, although testicular metastases are extremely rare, medical oncologists need to be cognizant that not all radiographic findings of 'physiologic uptake' in the testicles on PET scans should be concluded as such. Instead, the possibility of a testicular metastasis must be kept in mind at all times, particularly in patients who are beyond the fourth decade of life.

\section{References}

1 Casciato DA: Manual of Clinical Oncology, ed 6. Los Angeles, Wolters Kluwer/Lippincott Williams \& Wilkins, 2009.

2 Uchida K, Kurimura Y, Miyake M, Ando M, Tanaka T, Sasao T, Sano M, Yoshioka M, Yamaguchi Y: Testicular metastasis from squamous cell carcinoma of the lung. Int J Urol 2003;10:350-352.

-3 Kaplan MA, Kucukoner M, Inal A, Urakci Z, Firat U, Komek H, Isidogan A: Testicular mass: an initial sign of squamous cell carcinoma of the lung. World J Oncol 2012;3:291-293.

4 Grignon DJ, Shum DT, Hayman WP: Metastatic tumours of the testes. Can J Surg 1986;29:359-331.

5 Patel SR, Richardson RL, Kvols L: Metastatic cancer to the testes: a report of 20 cases and review of the literature. J Urol 1989;142:1003-1005.

6 Garcia-Gonzalez R, Pinto J, Val-Bernal JF: Testicular metastases from solid tumors: an autopsy study. Ann Diagn Pathol 2000;4:59-64.

7 Rosser CJ, Gerrard E: Metastatic small cell carcinoma to the testis. South Med J 2000;93:72-73.

8 Meares EM, Ho TL: Metastatic carcinomas involving the testis: a review. J Urol 1969;102:465-468.

-9 Palmieri D, Chambers AF, Felding-Habermann B, Huang S, Steeg PS: The biology of metastasis to a sanctuary site. Clin Cancer Res 2007;13:1656-1662.

10 Tummala MK, Hausner PF, McGuire WP, Gipson T, Berkman A: Case 1. Testis: a sanctuary site in merkel cell carcinoma. J Clin Oncol 2006;24:1008-1014.

11 Dhiren DS, Leppert JT, Rajfer J: Is the testis a chemo-privileged site? Is there a blood-testis barrier? Rev Urol 2007;9:28-32.

12 Riccardi R, Vigersky RA, Barnes S, Blever WA, Poplack DG: Methotrexate levels in the interstitial space and seminiferous tubule of rat testis. Cancer Res 1982;42:1617-1619. 


\section{Case Reports in Oncology}

\begin{tabular}{l|l}
\hline \multicolumn{2}{l|}{ Case Rep Oncol 2015;8:133-137 } \\
\hline DOI: $10.1159 / 000380814$ & $\begin{array}{l}\text { C } 2015 \text { S. Karger AG, Basel } \\
\text { www.karger.com/cro }\end{array}$ \\
\hline
\end{tabular}

Buck et al.: Testicular Metastasis in a Case of Squamous Cell Carcinoma of the Lung
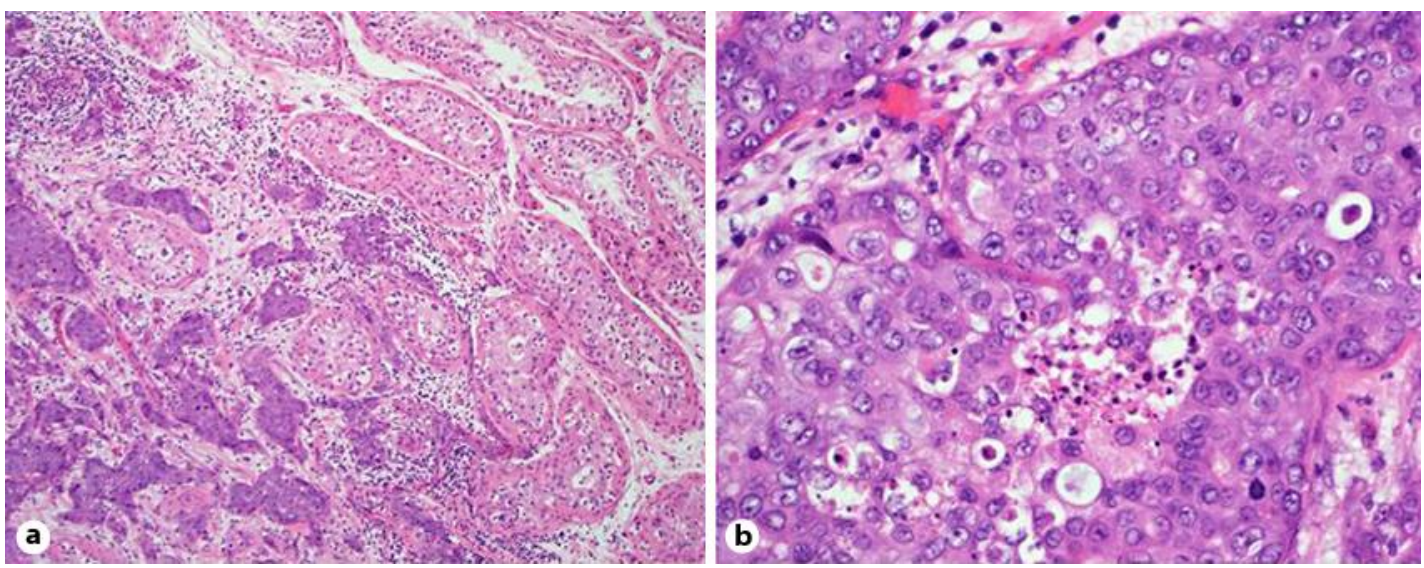

Fig. 1. Representative images of histopathology from the right radical orchiectomy. a The testis contained a well-circumscribed tumor measuring $1.5 \mathrm{~cm}$, no involvement of the spermatic cord, and negative surgical margins. The tumor showed anastomosing nests of large cells infiltrating between benign seminiferous tubules. HE. $\times 100$. b A higher-magnification image illustrates large cells with vesicular chromatin, large nucleoli, and abundant cytoplasm. HE. $\times 400$.
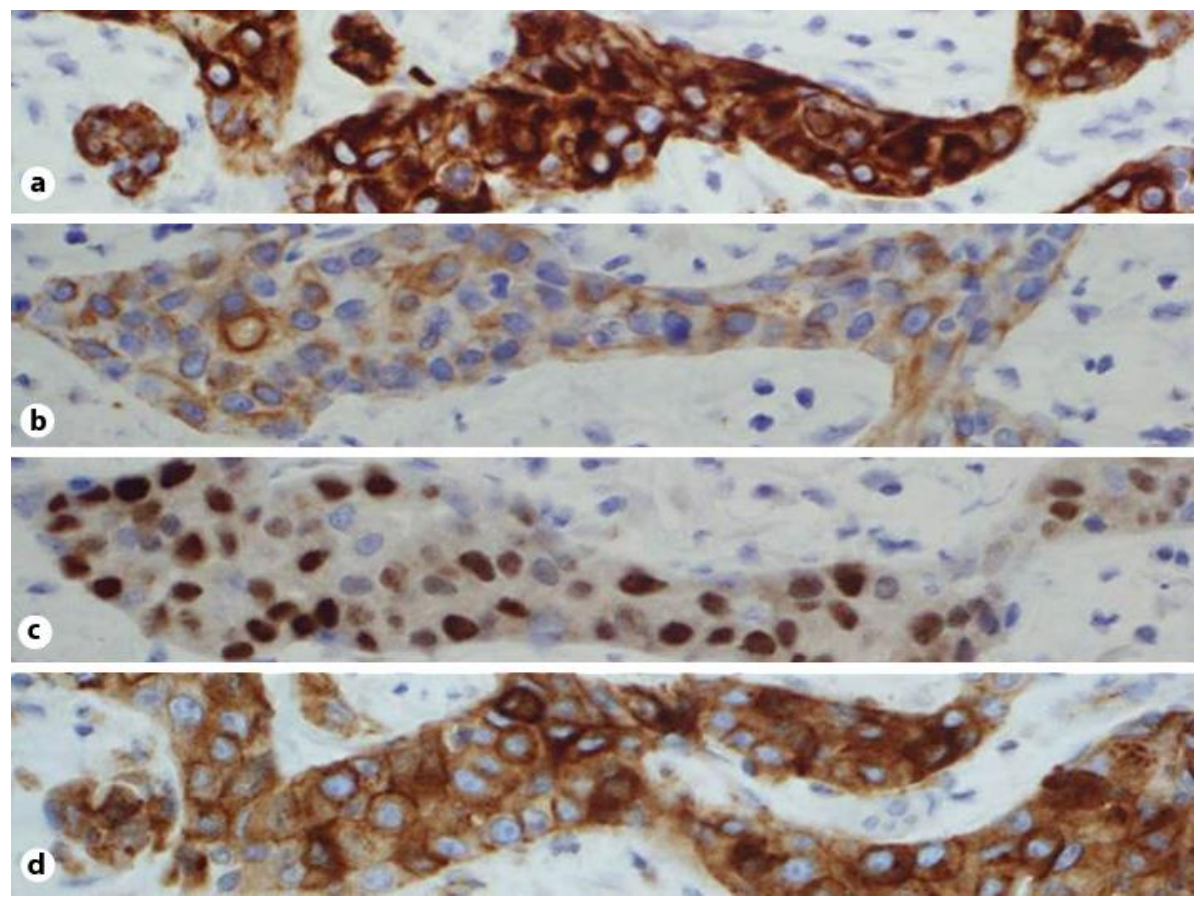

Fig. 2. Representative images of immunohistochemistry performed on the testicular mass. Immunoperoxidase stains show strong reactivity for pan-keratin (a), CK 5/6 (b), p63 (c), and polyclonal CEA (d). This phenotype supports the diagnosis of metastatic squamous cell carcinoma. $\times 200$. 